\title{
Experimental Realization of a Quaternary Bi-Chalcogenide Topological Insulator with Smaller Effective Mass
}

Pedro H. R. Goncalves ${ }^{1}$, Luan Calil', Igor Antoniazzi ${ }^{1}$, Thais Chagas ${ }^{1}$, Angelo Malachias ${ }^{1}$, Edmar A. Soares ${ }^{1}$, Vagner E. de Carvalho ${ }^{1}$, Douglas R. Miquita ${ }^{2}$, Rogério Magalhães-Paniago ${ }^{1,}{ }^{*}$ and Wendell S. Silva ${ }^{3}$

${ }^{1}$ Department of Physics, Federal University of Minas Gerais, Av. Antonio Carlos 6627, CEP 31270901, Belo Horizonte, Brazil

${ }^{2}$ Microscopy Center, Federal University of Minas Gerais, Av. Antonio Carlos 6627, CEP 31270901, Belo Horizonte, Brazil

${ }^{3}$ Brazilian Synchrotron Light Laboratory (LNLS), National Center for Research in Energy and Materials (CNPEM), CEP 13083-100, Campinas, Brazil

*Address to corresponding authors: $\underline{\text { rogerio@,fisica.ufmg.br }}$

We provide here, as supporting information, a detailed look on the SAED image, indicating more precisely the hexagonal lattice character of our sample (Fig. S1). The complete set of XPS spectra used for our analysis is shown in Fig. S2. The complete set of WDS spectra used for the calculation of the bulk composition of our sample is shown in Fig. S3. An ARPES measurement of BiSbSe $_{2.5} \mathrm{Te}_{0.5}$ using energy of $21.2 \mathrm{eV}$ from a He lamp is shown in Fig. S4, evidencing the surface nature of the linear states below the Fermi level and the same values of $\mathrm{k}_{\mathrm{F}}, \mathrm{E}_{\mathrm{D}}, \mathrm{v}_{\mathrm{g}}$ and $\mathrm{m} *$ obtained from the synchrotron ARPES measurements with energy of $103.5 \mathrm{eV}$. 
The Wavelenght Dispersion Spectroscopy (WDS) results were obtained at a JEOL JXA 8900RL model - WD/ED Combined Microanalyzer, operating at $15 \mathrm{kV}$. In a Johann optics arrangement the analyzing crystals used to cover the elements range were Lithium fluoride 200 $(\mathrm{LIF})-2 \mathrm{~d}=4.028$, Thallium acid pthalate $1011(\mathrm{TAP})-2 \mathrm{~d}=25.75$ and Pentaerythritol 002 (PET) $-2 \mathrm{~d}=8.742$.

The ARPES experiments of Fig. S4 were performed on an UHV system equipped with a SPECS PHOIBOS 100 spherical analyzer. The experiment was carried out at room temperature $(300 \mathrm{~K})$ with a base pressure better than $6.0 \times 10^{-10}$ using constant energy of $21.2 \mathrm{eV}$ from a $\mathrm{He}$ Lamp with energy and angular resolution better than $0.10 \mathrm{eV}$ and $0.2^{\circ}$, respectively.
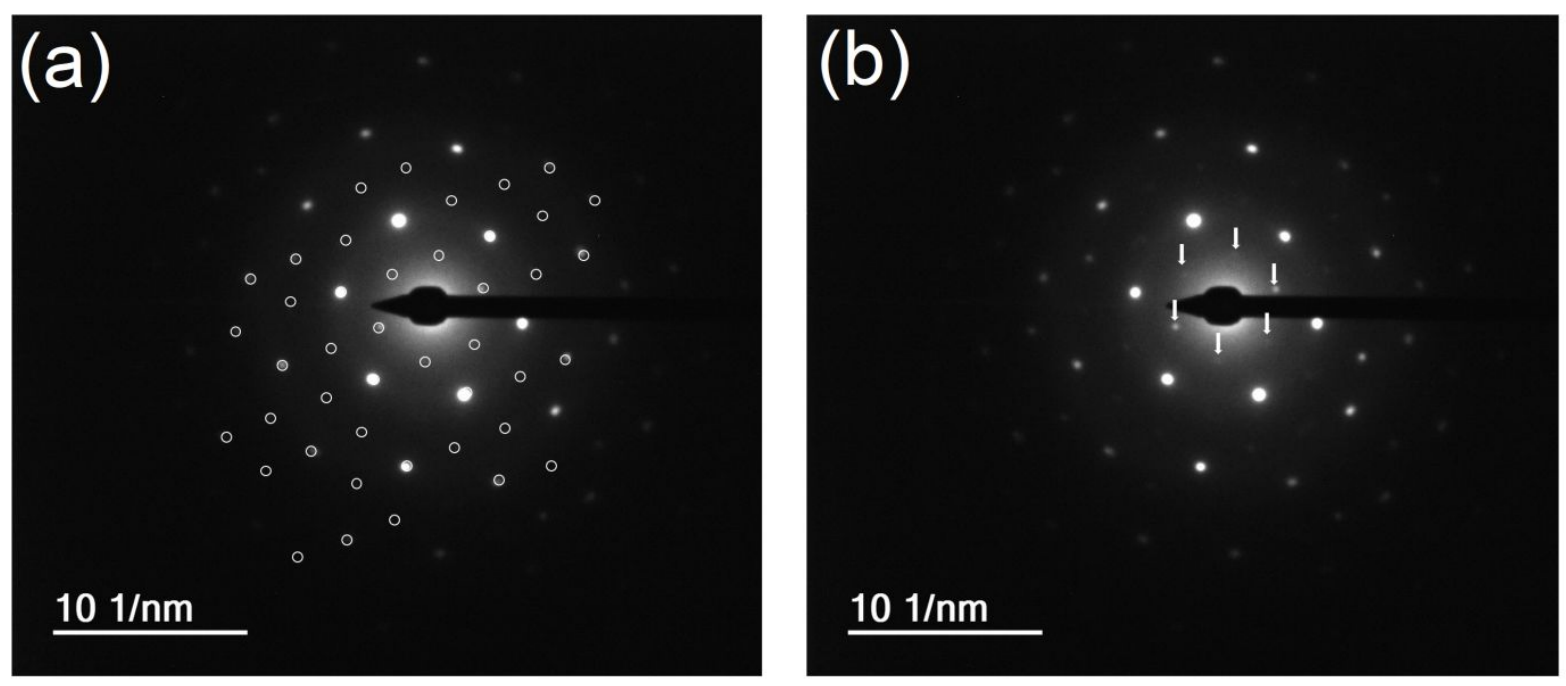

Fig. S1 - Detail of the SAED from fig 1(c). (a) Construction showing the hexagonal symmetry of the sample. (b) Detail on the perfect hexagonal lattice of the sample. White arrows show the $1^{\text {st }}$ order diffraction point which are obfuscated by the incident beam. 


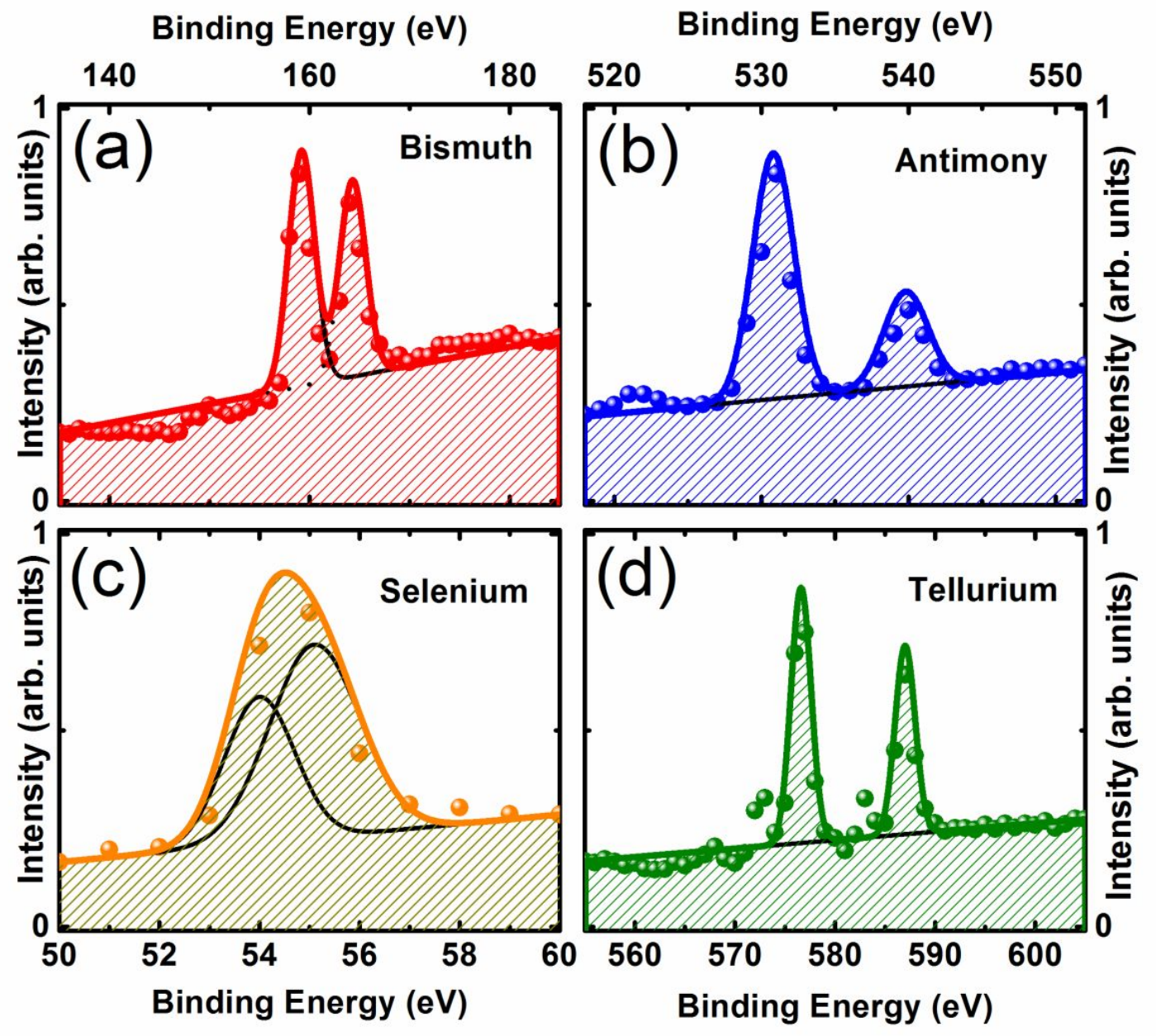

Fig. S2 - Analysis on the XPS spectra of (a) Bismuth 4f, (b) Antimony 3d, (c) Selenium 3d and (d) Tellurium 3d. The dots represent the data and the solid lines represent the fitting used for the composition calculation. 

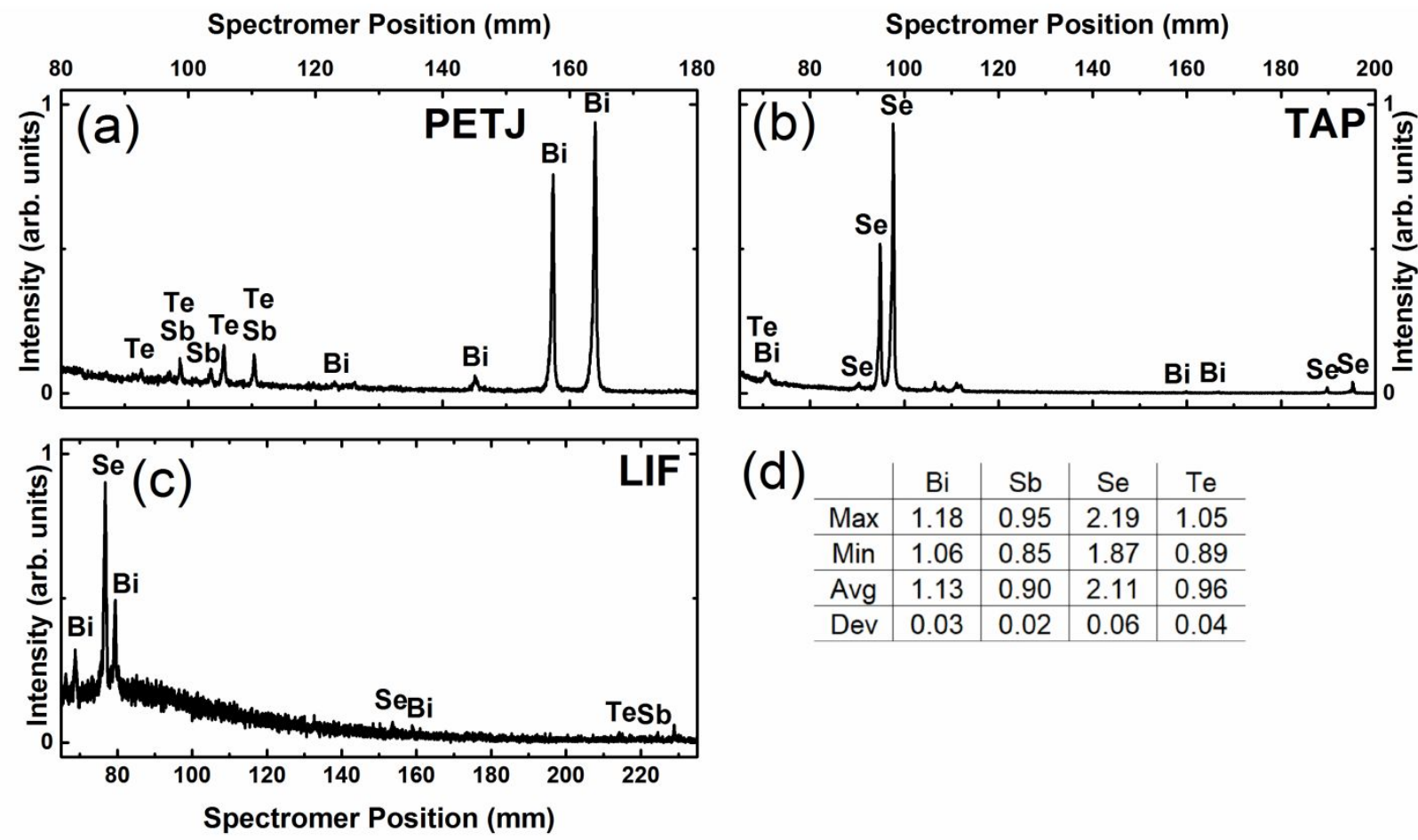

(d) \begin{tabular}{c|c|c|c|c} 
& $\mathrm{Bi}$ & $\mathrm{Sb}$ & $\mathrm{Se}$ & $\mathrm{Te}$ \\
\hline Max & 1.18 & 0.95 & 2.19 & 1.05 \\
\hline Min & 1.06 & 0.85 & 1.87 & 0.89 \\
\hline Avg & 1.13 & 0.90 & 2.11 & 0.96 \\
\hline Dev & 0.03 & 0.02 & 0.06 & 0.04
\end{tabular}

Fig. S3 - Analysis on the WDS spectra taken from (a) PETJ, (b) TAP and (d) LIF. (c) Table showing the values obtained for the concentration of each element. The values of average, minimum, maximum and standard deviation were taken from 30 points in the crystal. 


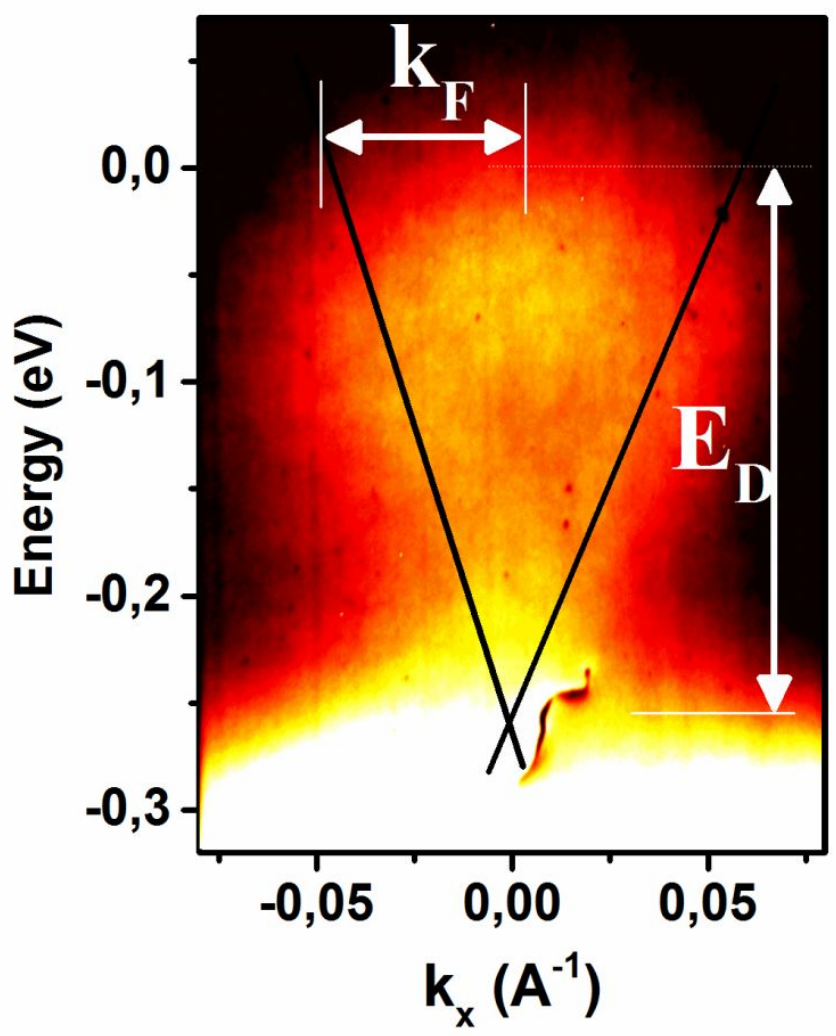

Fig. S4 - ARPES measurement of $\mathrm{BiSbSe}_{2.5} \mathrm{Te}_{0.5}$ with energy of $21.2 \mathrm{eV}$ provided by a He lamp. The values of $\mathrm{k}_{\mathrm{F}}=(0.05 \pm 0.01) \AA^{-1}$ and $\mathrm{E}_{\mathrm{D}}=(0.25 \pm 0.03) \mathrm{eV}$ were determined. They lead to an effective mass of $(0.08 \pm 0.04) \mathrm{m}_{\mathrm{e}}$ in agreement with ARPES measurements with $103.5 \mathrm{eV}$. 